\section{Gfi1 functions downstream of Math1 to control intestinal secretory cell subtype allocation and differentiation}

\author{
Noah F. Shroyer, ${ }^{1,2}$ Deeann Wallis, 1,3,6 \\ Koen J.T. Venken, ${ }^{4}$ Hugo J. Bellen, ${ }^{1,3,4,5}$ and \\ Huda Y. Zoghbi ${ }^{1,2,3,4,5,7}$
}

Departments of ${ }^{1}$ Molecular and Human Genetics, ${ }^{2}$ Pediatrics, and ${ }^{5}$ Neuroscience, ${ }^{3}$ Howard Hughes Medical Institute, ${ }^{4}$ Program in Developmental Biology, Baylor College of Medicine, Houston, Texas 77030, USA

Gfi1 is a transcriptional repressor implicated in lymphomagenesis, neutropenia, and hematopoietic development, as well as ear and lung development. Here, we demonstrate that Gfi1 functions downstream of Math1 in intestinal secretory lineage differentiation. $\mathrm{Gfi1}^{-/-}$mice lack Paneth cells, have fewer goblet cells, and supernumerary enteroendocrine cells. $\mathrm{Gfi1}^{-/-}$mice show gene expression changes consistent with this altered cell allocation. These data suggest that Gfi1 functions to select goblet/Paneth versus enteroendocrine progenitors. We propose a model of intestinal cell fate choice in which $\beta$-catenin and $C d x$ function upstream of Math1, and lineage-specific genes such as Ngn3 act downstream of Gfi1.

Supplemental material is available at http://www.genesdev.org.

Received July 11, 2005; revised version accepted August 8, 2005.

The epithelium of the small intestine is a highly proliferative tissue composed of four distinct cell types: absorptive enterocytes and three secretory lineages consisting of mucus-secreting goblet cells, hormone-secreting enteroendocrine cells, and antimicrobial peptide-secreting Paneth cells. All of these cell types derive from multipotent stem cells residing near the base of the Crypts of Liberkühn, the proliferative compartment of the intestinal epithelium (Madara and Trier 1994).

Differentiation within the crypts follows a spatial distribution: The stem cells are located near the base of the crypts and give rise to daughter cells that migrate up as they proliferate. Near the top of the crypt, these daughter cells terminally differentiate into the four main cell

[Keywords: Intestinal mucosa; developmental gene expression regulation; cell differentiation; Paneth cell; goblet cell; enteroendocrine cell] ${ }^{6}$ Present address: Deeann Wallis Schultz, National Human Genome Research Institute-Contractor, National Institutes of Health, 35 Convent Drive-MSC 3717, Building 35, Room 1B-407, Bethesda, MD 20892-3717, USA.

${ }^{7}$ Corresponding author.

E-MAIL hzoghbi@bcm.tmc.edu; FAX (713) 798-8728.

Article and publication are at http://www.genesdev.org/cgi/doi/10.1101/ gad. 1353905 . types of the intestinal epithelium. Absorptive enterocytes, goblet, and enteroendocrine cells migrate up the villus, whereas Paneth cells migrate down to reside at the crypt base.

The molecular mechanisms that underlie crypt formation and intestinal cell fate specification remain incompletely defined. Several genes have been implicated in crypt morphogenesis and proliferation, including Wnt $/ \beta$ catenin pathway members and target genes such as $C d x 1$ and $C d x 2$ (Korinek et al. 1998; Beck et al. 1999; Soubeyran et al. 1999). $\beta$-Catenin signaling is also necessary for stem cell renewal, proliferation, and differentiation (Pinto et al. 2003). The current model of intestinal epithelial differentiation suggests that $\beta$-catenin drives production of a pool of multipotent progenitors that use Notch signaling to select between Math1 or Hes1 expression (Yang et al. 2001; Sancho et al. 2004). Progenitors that express Hes1 will differentiate into absorptive enterocytes, whereas progenitors that express Math1 are committed to the secretory lineage and thus fated to become goblet, Paneth, or enteroendocrine cells. Additional transcription factors such as $N g n 3, P d x 1$, and Neurod1 are required for terminal differentiation of enteroendocrine cells (Schonhoff et al. 2004). The mechanism of selection between enteroendocrine, goblet, and Paneth cells remains unclear, and additional factors are hypothesized to direct differentiation of goblet and Paneth cells.

Math1 is a basic helix-loop-helix (bHLH) transcription factor important in cell fate determination (Akazawa et al. 1995; Ben-Arie et al. 1997). Math1-null embryos die at birth due to respiratory failure and lack many specific cell lineages, including cerebellar granule neurons, spinal cord interneurons, inner ear hair cells, and intestinal secretory cells (Ben-Arie et al. 1997; Bermingham et al. 1999, 2001; Yang et al. 2001).

Gfil is a zinc-finger transcriptional repressor essential for hematopoietic stem cell function and differentiation of immune cells and a proto-oncogene that is a frequent target of retroviral insertions in lymphoid tumors (Schmidt et al. 1996; Karsunky et al. 2002; Hock et al. 2003, 2004). Recently, Gfi1 was shown to be essential for formation of lung neuroendocrine cells and may cooperate with Mash1 to promote neuroendocrine lung tumors (Kazanjian et al. 2004). In the inner ear, Gfi1 is dependent on Math1 for its expression, and is important for both differentiation and survival of the sensory hair cells (Wallis et al. 2003). Thus, Gfil functions downstream of and cooperates with Notch-regulated bHLHs (Math1 and Mash1) to promote differentiation of bHLH-specified cell types, similar in function to its Drosophila homolog senseless (Jafar-Nejad et al. 2003; Jafar-Nejad and Bellen 2004). Here we show that Gfil functions downstream of Math1 in the intestinal epithelium, where it functions to select Paneth/goblet versus enteroendocrine cell fates among intestinal secretory progenitors.

\section{Results and Discussion}

GFI1 is expressed in the secretory lineage of the intestine and is absent in Math $1^{\text {lacz/lacz }}$ mutant intestine

To identify genes regulated by Math1, we assessed gene expression in $\mathrm{Math}^{-/}$embryonic intestine using oligo- 
nucleotide microarrays and identified 192 genes that were significantly misexpressed in Math1-/- intestine (N.F. Shroyer and H.Y. Zoghbi, unpubl.). We next performed Northern blot and quantitative real-time RTPCR analysis of gene expression in Math1 $1^{\text {lacz/+ }}$ and Math1 1acZ/lacZ intestines from two embryonic stages, confirming significant expression changes in 11 genes (Supplementary Fig. 1; Supplementary Table 1). We observed reduced expression of secretory lineage-specific transcripts (i.e., Agr2, Neurod1, Ngn3, Pdx1, Sct, Spdef), in agreement with the proposed function for Math1 in intestinal secretory cell specification (Yang et al. 2001).

We found that Gfil expression was reduced 4.4- to 7.6-fold in Math1 1acZ/lacz embryonic intestine (Supplementary Table 1). Previously, we showed that Gfil mRNA is expressed in the developing intestine at embryonic day 12.5 (E12.5) (Wallis et al. 2003). To further investigate GFI1 expression in the intestine, we performed immunohistochemical analyses (Fig. 1). In wildtype mice, robust GFI1 expression can be detected at E14.5 in scattered cells throughout the developing intestinal epithelium (Fig. 1A). At E18.5, GFI1 is found primarily on the villus epithelium (data not shown). As expected from our microarray and real-time RT-PCR results, GFI1 protein is not detectable in Math1lacZ/lacZ epithelium (Fig. 1B). In the mature intestine, GFI1 is found in the small and large intestines, primarily in the crypt but also in some villus cells (Fig. 1C,D).

To determine which cell types within the intestinal epithelium express GFI1, we performed double-labeling experiments with cell-type-specific markers. We performed confocal immunofluorescence with antibodies for GFI1 and $\beta$-galactosidase in intestines from adult Math1 $1^{\text {lacz/+ }}$ mice (Fig. 1E-G). In Math1 $1^{\text {lacZ/+ }}$ mice, $\beta$-galactosidase acts as a lineage marker for cells that have expressed MATH1 (Ben-Arie et al. 2000); we find that

Figure 1. GFI1 expression in intestine. $(A-D)$ Immunohistochemistry for GFIl protein, in brown. (A) E14.5 wild-type (wt) intestine. GFI1 is expressed throughout the developing intestinal epithelium. (B) E14.5 Math1 1acZ/lacZ intestine. GFI1 expression is absent. $(C, D)$ GFI1 expression in adult ileum and colon. GFI1 is expressed in the crypt and villus in both small intestine and colon (arrowheads). $(E-G)$ Confocal images of a crypt from Math1 $1^{1 a c Z /+}$ ileum showing colocalization of GFI1 and the Math $1^{\text {1acZ }}$ product $\beta$-galactosidase. $(E)$ GFI1 (red). (F) $\beta$-Galactosidase (green). ( $G)$ Merge of $E$ and $F$ with TOTO-3 DNA stain (blue). A cell expressing both GFI1 and $\beta$-galactosidase is indicated by the white arrowhead. $(H)$ Colocalization of MATH1 and GFI1 in an ileal crypt. GFI1 (red) and MATH1 (green) are coexpressed. $(I, J)$ Serial sections showing colocalization of GFI1 and NGN3. Three-micrometer serial sections were stained for GFI1 $(I)$ or NGN3 $(J)$. A cell coexpressing both proteins is indicated by the arrow. A cell expressing only GFIl protein is shown by the arrowhead. $(K-M)$ Colocalization of GFI1 and CgA. Low-power $(K)$ and high-power $(L)$ images of wild-type ileum stained for GFI1 (brown) and CgA (blue). Cells expressing only CgA are indicated by blue arrows; cells expressing only GFIl are indicated by the brown arrowheads; cells expressing both $\mathrm{CgA}$ and GFIl are indicated by black arrowheads. $(M)$ Confocal images of crypts from wild-type ileum showing colocalization of GFI1 and CgA. A merged image similar to $G$ is shown, with TOTO-3 in blue, GFI1 in red, and CgA in green. The arrowheads show cells that coexpress GFIl and CgA. $(N)$ Colocalization of GFI1 (green) with serotonin (5HT, red). Cells expressing both GFIl and serotonin are indicated by white arrowheads; a cell expressing only GFI1 is indicated by the green arrow. (O) No colocalization of anti-GFI1 (brown) and alcian blue (AB; blue), a marker for goblet cells, in wild-type adult jejunum. $(P)$ No colocalization of anti-GFI1 (brown) and anti-lysozyme (Lys; purple), a marker for Paneth cells, in wild-type adult ileum.
GFI1 is expressed in a subset of these secretory progenitors (Fig. 1G). Colabeling for both GFI1 and MATH1 proteins identified coexpression in a subset of crypt progenitors (Fig. $1 \mathrm{H})$. We also found coexpression of GFI1 with NGN3, a marker of enteroendocrine precursors (Fig. 1I,J). Thus, within the crypt, GFI1 is expressed in a subset of secretory progenitors that includes enteroendocrine precursors.

Expression of GFI1 on villi (Fig. 1C) suggested that GFI1 is also expressed in some mature epithelial cells. We performed colabeling studies utilizing chromogranin $\mathrm{A}(\mathrm{CgA})$ as a panendocrine marker (Fig. $1 \mathrm{~K}-\mathrm{M})$, alcian blue to mark goblet cells (Fig. 1O), and lysozyme to mark Paneth cells (Fig. 1P). We found that GFI1 expressing cells colabeled with CgA in both crypts and villi (black arrowheads in Fig. $1 \mathrm{~K}, \mathrm{~L}$ ), whereas we were unable to find GFI1 expression in alcian blue- or lysozyme-positive cells (Fig. 1O,P). This indicates that GFI1 is expressed in enteroendocrine cells, but not goblet or Paneth cells. Immunofluorescence microscopy confirmed colocalization of GFI1 and CgA (Fig. 1M). Quantification showed that among villus cells expressing CgA or GFI1, 50\% coexpress GFI1 and CgA, 10\% express GFI1 only, and 40\% express CgA only; within the crypt, 60\% coexpress GFI and CgA, 20\% express GFI1 only, and 20\% express CgA only. Analysis of GFI1 coexpression with the secretory products of mature endocrine cells showed that GFI1 is coexpressed with serotonin (Fig. 1N) but we detected no coexpression with PYY, Neurotensin, Secretin, nor CCK producing enteroendocrine subsets (data not shown). Together these results show that GFI1 is expressed within

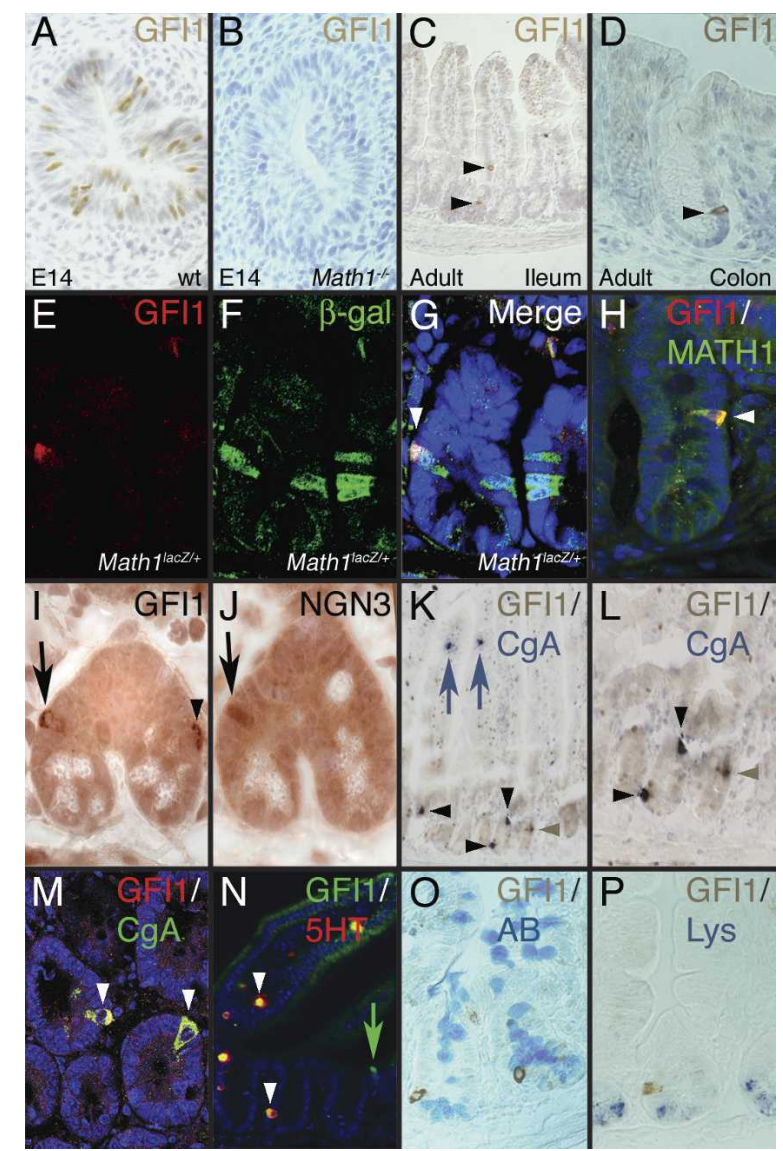


Shroyer et al.

the crypt in Math1-dependent progenitor cells, including NGN3-expressing enteroendocrine precursors, as well as a subset of enteroendocrine cells on the villus.

\section{Gfil mutant mice have abnormal secretory lineage production}

Based on the expression pattern of GFI1 in the intestinal epithelium, we hypothesized that $G f_{i 1}{ }^{-/-}$mice may have differentiation defects. The overall crypt-villus structure of the intestine from $G \mathrm{fil}^{-/-}$mice is normal, including the presence of Peyer's patches (Fig. 2; Supplementary Fig. 2; data not shown). However, we found an overt

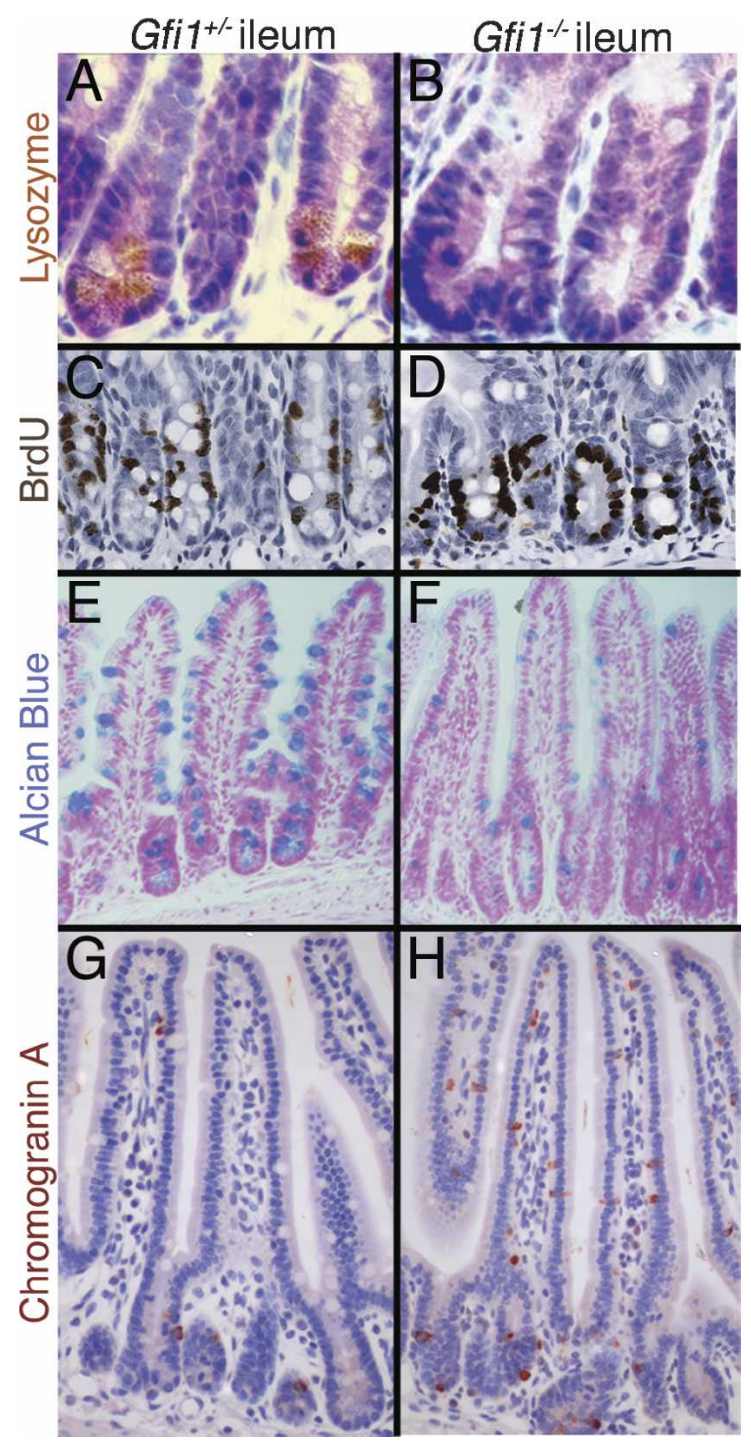

Figure 2. Gfil is required for secretory cell lineage specification. $(A, B)$ Anti-lysozyme staining (brown) shows a lack of immunoreactivity in $G f_{11^{-1}}$ ileum, suggesting the absence of Paneth cells. $(C, D)$ BrdU labeling of proliferating cells following a 2-h BrdU pulse. (C) In wild-type ileum, proliferating cells are excluded from the Paneth cell zone at the base of the crypt. (D) In $G f i 1^{-/-}$ileum, proliferating cells are found throughout the crypt base, consistent with an absence of the Paneth cell zone. $(E, F)$ Alcian blue staining is reduced in Gfi1 ${ }^{-/}$ileum $(F)$ compared with wild-type littermates $(E) .(G, H)$ Anti-CgA immunohistochemistry marks more cells in $\mathrm{Gfi1}^{-{ }^{-}}$ileum $(H)$ than wild-type littermates $(G)$. reduction in the number of secretory cells. Gfi1 ${ }^{-/-}$mice have no apparent Paneth cells, as determined by hematoxylin and eosin, alcian blue, periodic acid Schiff/alcian blue, and anti-lysozyme staining (Fig. 2A,B; data not shown). In wild-type crypts, proliferating cells are largely excluded from the Paneth cell zone at the base of the crypt (Cheng and Leblond 1974). Two hours following injection with BrdU, labeled cells are primarily found above the crypt base in $\mathrm{Gfi}^{+/-}$mice (Fig. 2C), whereas in Gfi1 ${ }^{-/-}$mice, BrdU-labeled cells are observed throughout the crypt base (Fig. 2D), consistent with the apparent absence of Paneth cells. Gfi1 ${ }^{-1-}$ mice had approximately one-third the normal number of goblet cells throughout the small intestine $(p<0.0001)$, as shown by alcian blue staining (Figs. 2E,F, 3). This reduction in goblet cell number was also observed in the colon (Supplementary Fig. 2C,D). In contrast to goblet and Paneth cells, we observed a significant increase in the number of enteroendocrine cells in $\mathrm{Gfi1}^{-/-}$mice (Fig. 2G,H; Supplementary Fig. 2E,F), with 3.6-fold more enteroendocrine cells across the small intestine as determined by CgA immunohistochemistry $(p<0.0001)$ (Fig. 3$)$, as well as a significant increase $(p<0.0001)$ in all enteroendocrine subtypes examined across the small intestine and colon /secretin, fivefold increase in jejunum; neurotensin, eightfold increase in ileum; CCK, fourfold increase in jejunum; PYY, sixfold increase in colon) (Supplementary Fig. 31.

We next examined expression of known intestinal epithelial genes in $\mathrm{Gfil}^{-/}$intestine by real-time RT-PCR. As shown in Table 1, expression of genes specific to the four intestinal epithelial lineages reflects the lineage allocation defect in $\mathrm{Gfi1}^{-/-}$intestines. Tff3, encoding a goblet-specific trefoil factor, is reduced threefold across the intestine; Lyzs and Mmp7, encoding Paneth genes, are reduced 5.9- and 5.1-fold, respectively, in the ileum, where Paneth cells are most prominent; no change was detected in expression of the absorptive enterocyte-specific sucrase-isomaltase gene. We analyzed several genes known to be important for enteroendocrine specification and differentiation, including Islet1, Neurod1, and Ngn3. As expected from the increase in enteroendocrine cell numbers, these genes showed significantly increased expression in $\mathrm{Gfi1}^{-/-}$intestines (Table 1). Thus, loss of Gfil results in a reduction of the RNA levels of goblet- and Paneth-specific genes and an increase in the RNA levels of genes important for the differentiation of enteroendocrine cells. These results provide strong evidence that Gfil is essential for normal lineage allocation in the intestine, and suggest that it functions in early crypt progenitors prior to cell-specific differentiation genes such as Ngn3, Neurod1, and Isl1.

To determine whether the secretory lineage abnormalities in $\mathrm{Gfil}^{-/-}$mice were due to abnormal proliferation or apoptosis, we performed anti-Ki67, anti-BrdU staining after a $2-\mathrm{h}$ BrdU pulse and TUNEL staining. No significant alteration in Ki67, BrdU, or TUNEL staining was observed (data not shown), indicating an overall normal production of epithelial cells and no evidence of increased apoptosis of crypt progenitors. Although our data cannot exclude the possibility that GFI1 might regulate proliferation of a subset of progenitor cells within the crypt, the data are consistent with a change in secretory progenitor cell fate in $\mathrm{Gfi1}^{-/-}$mice from goblet/Paneth to enteroendocrine. We tested this possibility by examining secretory lineage fate in $\left[\mathrm{Math}^{1 \mathrm{lacZ/+}} ; \mathrm{Gfi1}^{-/-}\right]$mice. By 


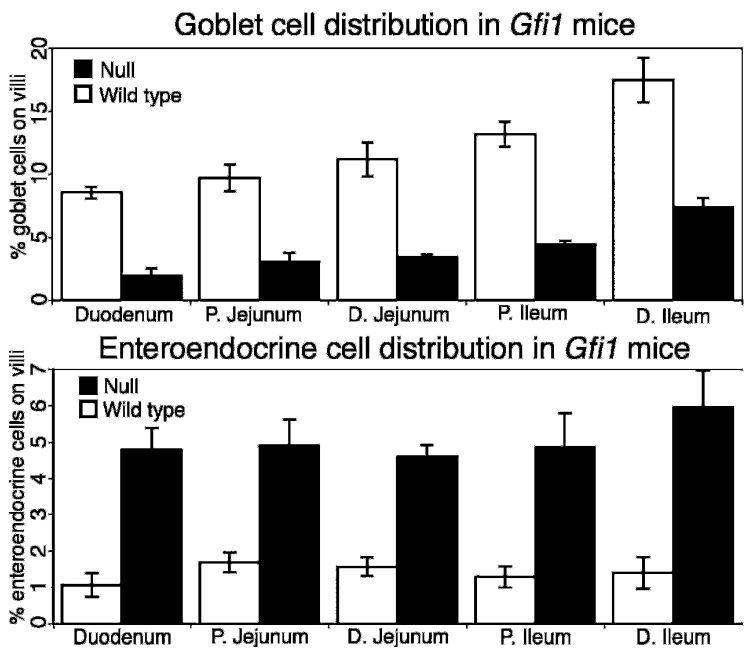

Figure 3. Quantification of goblet and enteroendocrine cells in $\mathrm{Gfi1}^{-/}$mice. (Top) Quantification of goblet cells, derived from counting alcian blue-positive cells. Cell numbers are graphed as a percentage of total villus epithelial cells. Open bars represent $\mathrm{Gfi1}^{+/}$, black bars represent $\mathrm{Gfi1}^{-/}$, and error bars represent the standard error $(N=$ four animals per genotype). Subregions of the small intestine are indicated on the $X$-axis. (P) proximal; (D) distal. (Bottom) Quantification of enteroendocrine cells, derived from counting CgA-positive cells; graphs are shown as above.

following the fate of $\beta$-galactosidase-positive cells in these $G \mathrm{fil}^{-/-}$mice, we show that all MATH1-expressing cells retain their commitment to the secretory fate and do not switch to an absorptive enterocyte fate (Fig. 4A). Thus, we conclude that Paneth and goblet progenitors adopt an enteroendocrine fate in the absence of GFI1.

\section{Pathway analysis for secretory cell production}

To position Math1 and Gfi1 in the genetic hierarchy of intestinal specification, we examined the expression of genes known to be important for intestinal epithelial differentiation by real-time RT-PCR. Analyses of experimentally induced intestinal metaplasias suggest that $\beta$-catenin and $C d x 2$ can induce Math1 and Gfi1 (Mutoh et al. 2002; Okubo and Hogan 2004). We found that $\beta$-catenin target genes $C d \times 2, E p h b 2$, and Ephb3 (van de Wetering et al. 2002) are unchanged in Math1lacZ/lacZ embryonic intestine (Supplementary Table 1), supporting the hypothesis that Math1 functions downstream of $\beta$-catenin and $C d \times 2$, and consistent with our hypothesis that Math1 is expressed at an early progenitor stage in crypt differentiation in cells that are selecting between absorptive and secretory fates (Yang et al. 2001). In $\mathrm{Gfi}^{-/-}$intestine we found up-regulated expression of Ephb2 and Ephb3 (Table 1) and continued expression of EPHB3 protein at the crypt base in the absence of mature Paneth cells (data not shown). EPHB3, thought to be Paneth cell-specific, and EPHB2, expressed in proliferating crypt progenitors, are important for vectorial migration of differentiated cells within and out of the crypt-in their absence migration is aberrant and Paneth cells are displaced (Batlle et al. 2002). Our results suggest that expression of Ephb2 and Ephb3 is not dependent upon differentiation of secretory cells, and that crypt morphogenesis and directional migration is independent of normal cellular specification. Further, Ephb3 may not be unique to Paneth cells as suggested by van Es et al.
(2005), but rather to cells at the crypt base regardless of identity. Thus, these $\beta$-catenin targets may be expressed independently of Paneth cell maturation in $\mathrm{Gfil}^{-/-}$intestine.

Other genes previously implicated in intestinal epithelial differentiation (EfnB1, EfnB2, Klf5, Cdx2, Elf3/Ets1) are unchanged in both $G f i 1^{-1-}$ and Math1 $1^{\text {lacZ/lacZ }}$ intestines. These results suggest that $C d \times 2, E l f 3$, and Klf5 are upstream or independent of Math1 and Gfil. We found a 1.4- to 2.4-fold reduction in Math1 expression in $\mathrm{Gfi1}^{-/-}$ intestine, consistent with the overall reduction in secretory cells (which normally continue to express Math1 as they migrate up the villus); this result was confirmed by immunohistochemistry with anti-MATH1 antibodies that show continued expression of MATH1 protein in Gfi1 ${ }^{-/-}$intestines (Table 1; data not shown). This continued expression at near normal levels demonstrates that Math1 is not dependent upon Gfi1 for its expression, although these data do not exclude the potential for GFI1 to regulate Math1 levels. Klf4, required for normal goblet cells and absorptive enterocytes (Katz et al. 2002), is slightly down-regulated in both Math1 $1^{\text {lacZ/lacZ }}$ and $G f i 1^{-1-}$ mice, likely reflecting the loss of goblet cells in these mice. Hes1 is unchanged in $\mathrm{Gfi1}^{-/-}$, consistent with normal Notch-based selection of secretory versus absorptive progenitors in Gfil mutant mice. We propose an extended model for differentiation of intestinal secretory cells: Stem cell renewal and normal differentiation is dependent upon Wnt/ $\beta$-catenin signaling and $C d x$ genes, specification of a secretory-specific progenitor is dependent on Math1, and selection between goblet/Paneth and enteroendocrine progenitors is dependent on Gfi1 (Fig. 4B). Consistent with this model are findings from studies of $\mathrm{Ngn3}^{-/-}$mice that revealed a balance between production of enteroendocrine and goblet cells

Table 1. Gene expression changed in $\mathrm{Gfil}^{-/-}$

\begin{tabular}{lccrc}
\hline Gene & Jejunum & Ileum & Colon & Overall \\
\hline Cdx2 & $1.1^{\mathrm{ns}}$ & $1.1^{\mathrm{ns}}$ & $1.0^{\mathrm{ns}}$ & $1.1^{\mathrm{ns}}$ \\
EfnB1 & $1.1^{\mathrm{ns}}$ & $1.1^{\mathrm{ns}}$ & $-1.1^{\mathrm{ns}}$ & $1.0^{\mathrm{ns}}$ \\
EfnB2 & $1.6^{\mathrm{ns}}$ & $1.3^{\mathrm{ns}}$ & $-1.4^{\mathrm{ns}}$ & $1.2^{\mathrm{ns}}$ \\
Elf3 & $1.7^{\mathrm{ns}}$ & $1.2^{\mathrm{ns}}$ & $-1.1^{\mathrm{ns}}$ & $1.3^{\mathrm{ns}}$ \\
Ephb2 & $2.8^{\mathrm{b}}$ & $2.3^{\mathrm{b}}$ & $2.6^{\mathrm{ns}}$ & $2.6^{\mathrm{d}}$ \\
Ephb3 & $2.1^{\mathrm{a}}$ & $1.5^{\mathrm{ns}}$ & $1.9^{\mathrm{ns}}$ & $1.8^{\mathrm{b}}$ \\
Hes1 & $1.4^{\mathrm{ns}}$ & $1.1^{\mathrm{ns}}$ & $-1.5^{\mathrm{ns}}$ & $1.1^{\mathrm{ns}}$ \\
Isl1 & $7.9^{\mathrm{c}}$ & $13.4^{\mathrm{c}}$ & $6.3^{\mathrm{b}}$ & $9.2^{\mathrm{d}}$ \\
Klf4 & $-1.2^{\mathrm{ns}}$ & $-1.2^{\mathrm{ns}}$ & $-1.4^{\mathrm{a}}$ & $-1.3^{\mathrm{b}}$ \\
Klf5 & $1.4^{\mathrm{ns}}$ & $1.1^{\mathrm{ns}}$ & $-1.1^{\mathrm{ns}}$ & $1.1^{\mathrm{ns}}$ \\
Lyzs & $-1.5^{\mathrm{ns}}$ & $-5.9^{\mathrm{c}}$ & $1.3^{\mathrm{ns}}$ & $-1.4^{\mathrm{d}}$ \\
Math1 & $-2.1^{\mathrm{ns}}$ & $-2.4^{\mathrm{b}}$ & $-1.4^{\mathrm{ns}}$ & $-2.0^{\mathrm{c}}$ \\
Mmp7 & $-1.6^{\mathrm{ns}}$ & $-5.1^{\mathrm{b}}$ & $-4.6^{\mathrm{ns}}$ & $-3.8^{\mathrm{c}}$ \\
Neurod1 & $9.2^{\mathrm{b}}$ & $17.9^{\mathrm{b}}$ & $9.7^{\mathrm{a}}$ & $12.3^{\mathrm{d}}$ \\
Ngn3 & $6.2^{\mathrm{c}}$ & $8.1^{\mathrm{b}}$ & $16.3^{\mathrm{b}}$ & $10.2^{\mathrm{d}}$ \\
Pdxl & $-1.4^{\mathrm{ns}}$ & $8.1^{\mathrm{b}}$ & $1.0^{\mathrm{ns}}$ & $3.3^{\mathrm{ns}}$ \\
Si & $-1.4^{\mathrm{ns}}$ & $-1.3^{\mathrm{ns}}$ & $-2.3^{\mathrm{ns}}$ & $-1.7^{\mathrm{ns}}$ \\
Tff3 & $-3.0^{\mathrm{d}}$ & $-3.3^{\mathrm{d}}$ & $-3.2^{\mathrm{d}}$ & $-3.2^{\mathrm{d}}$ \\
\hline Vales & Mtion & &
\end{tabular}

Values are the ratio $\left(\mathrm{Gfi1}^{-/-} / \mathrm{Gfi1}^{+/-}\right)$of normalized expression levels. Negative values indicate reduced gene expression in $\mathrm{Gfi1}^{-/-}$intestines compared to wild type.

${ }^{\mathrm{a}} p<0.05$.

${ }^{\mathrm{b}} p<0.01$

${ }^{\mathrm{c}} p<0.001$.

$\mathrm{d}_{p}<0.0001$.

${ }^{\mathrm{ns}}$ Not significant. 

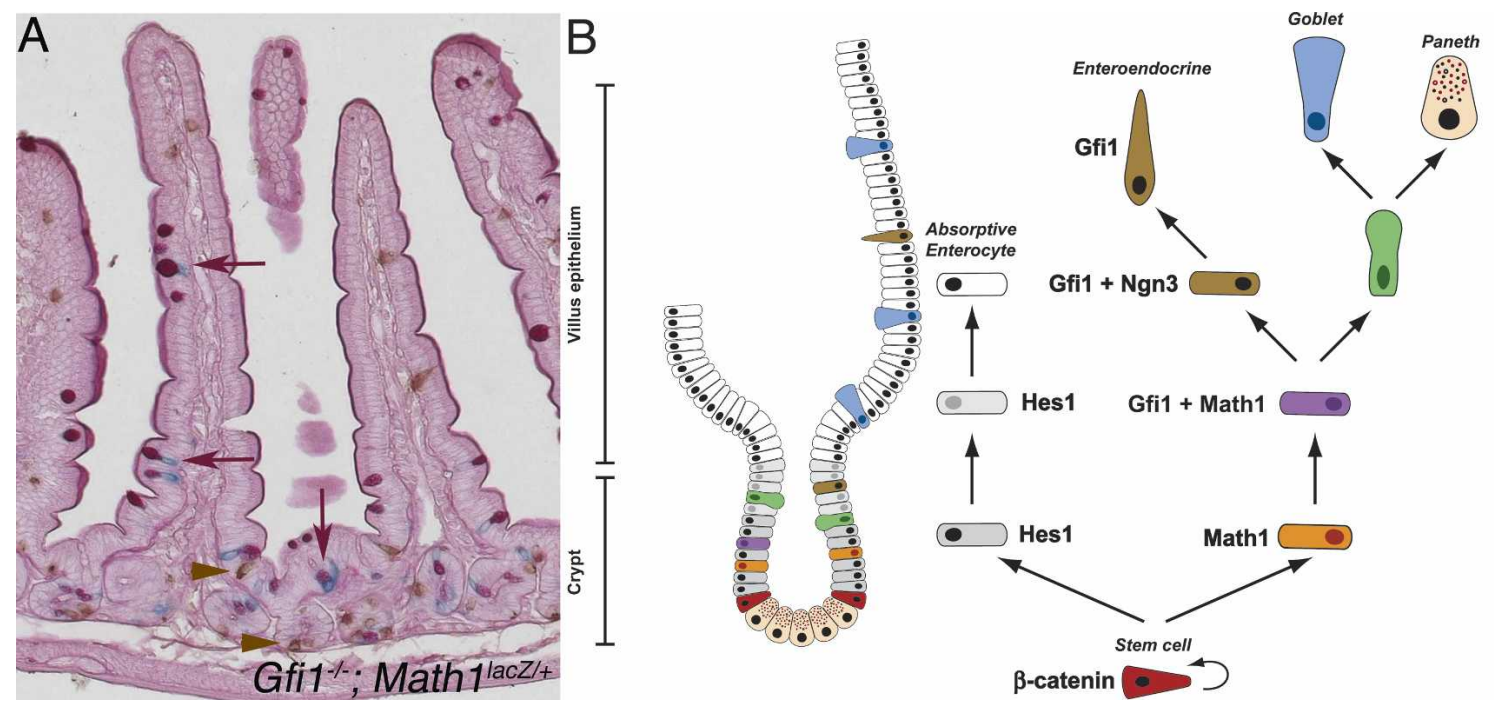

Figure 4. (A) Secretory cells remain lineage committed in $G$ fi $1^{-/-}$intestine. Ileum from $\left[\mathrm{Gfi}^{-1^{--}}\right.$; Math $\left.1^{1 a c z /+}\right]$ mice is shown. The intestine was stained with Xgal (blue) for cells that expressed $\beta$-galactosidase under the control of the Math1 locus to mark intestinal secretory cells, periodic acid-Schiff (pink) to mark goblet and Paneth cells, and anti-serotonin immunohistochemistry (brown) to mark enteroendocrine cells. All cells marked by Xgal are marked by periodic acid-Schiff (purple arrows) or serotonin (brown arrowheads), indicating secretory lineage restriction in $\mathrm{Gfi1}^{-/}$intestine. Note also the lack of periodic acid-Schiff-positive Paneth cells at the crypt base. $(B)$ Model for intestinal epithelial differentiation. (Left) A cartoon drawing of a crypt and adjoining villus epithelium is shown. (Right) A diagram detailing the cellular and molecular events in epithelial differentiation. Enteroendocrine, Paneth, goblet, and absorptive enterocytes are indicated by name. Self-renewing stem cells, shown in red, are located near the base of the crypt and show nuclear localization of $\beta$-catenin. Stem cells produce highly proliferating multipotent progenitors, which employ Notch signaling to select Hes1-expressing cells versus Math1-expressing cells. Hes1-expressing cells (gray) go on to differentiate into absorptive enterocytes (white), which constitute the majority of the mature epithelium. Math1-expressing cells (orange) constitute the secretory progenitors. These cells subsequently coexpress Gfi1 (purple), which functions to select between goblet/Paneth progenitors (green) and enteroendocrine precursors (brown). Enteroendocrine precursors express Ngn3 and Gfi1; Gfil continues to be expressed in a subset of mature enteroendocrine cells.

(Jenny et al. 2002). Our model proposes that GFI1 expression must be extinguished for normal differentiation of goblet/Paneth progenitors. Alternatively, GFI1 may be expressed only in MATH1/NGN3-positive enteroendocrine precursors that, in the absence of Gfil, are overproduced and inhibit production of goblet/Paneth cells from adjacent progenitors. Fate mapping studies of GFI1positive progenitors will distinguish these mechanisms.

In sum, we suggest that Gfil functions in a Math1specified, secretory lineage-committed progenitor to select between goblet/Paneth and enteroendocrine cell fates. These data are the first to demonstrate a step in secretory lineage allocation separating enteroendocrine and Paneth/goblet fates, and provide additional evidence to support the close ontogenic relationship between Paneth and goblet cells. That the oncogene Gfil is a key player in the proliferative intestinal crypt suggests a potential role for Gfil in intestinal malignancies. Further work to identify additional targets of Math1 and Gfi1 in the intestine will likely identify additional factors important for the differentiation of secretory lineages and clarify the molecular hierarchy of intestinal epithelial differentiation. Our findings support the concept that Gfil/senseless homologs cooperate with bHLH factors to direct cell fate decisions in multiple tissues, and that this genetic partnership may be utilized throughout the animal kingdom to specify cell fates.

\section{Materials and methods}

Additional materials and methods are available as Supplemental Material.
Animals

Gfi1 mutant and Math1 mutant mice were bred and genotyped as previously described (Ben-Arie et al. 2000; Hock et al. 2003).

Embryo collection, RNA extraction, and gene expression analysis Tissues from $\mathrm{Gfi1}^{-/-}$and $\mathrm{Gfi1}^{+/-}$animals and from Math1 $1^{1 a c Z /+}$ and Math1 1acZ/lacz embryos were dissected and stored in RNAlater (Ambion). RNA was extracted from intestines with Trizol (Invitrogen) followed by purification on RNeasy columns (Qiagen). RNA quality was assessed by formaldehyde-agarose gel electrophoresis and Bioanalyser 2100 (Agilent) analysis. For Northern blots, $5 \mu \mathrm{g}$ of total RNA was separated, transferred to a nylon membrane, and hybridized with the appropriate ${ }^{32} \mathrm{P}$ labeled DNA probe corresponding to each gene. Signal intensities were measured by densitometry and expression levels were normalized to Gapdh. For real-time RT-PCR, cDNA equivalent to $100 \mathrm{ng}$ of total RNA was PCR-amplified in an ABI PRISM 7300 detection system. The primers and probes for each gene analyzed are listed in Supplementary Table 2. The relative level of each RNA was calculated using the standard curve method and normalized to the corresponding Gapdh RNA levels. ANOVA and $t$-tests were performed to measure variations in gene expression between groups.

Tissue preparation and Immunohistochemistry

Tissues were dissected and fixed overnight in $4 \%$ paraformaldehyde, then embedded in paraffin for sectioning. Five-micrometer sections were collected and analyzed by immunohistochemistry. For serial sections, 3- $\mathrm{mm}$ sections were collected and alternate sections stained for GFIl or NGN3. For confocal microscopy, $12-\mu \mathrm{m}$ frozen sections were collected onto slides, post-fixed in formalin for $1 \mathrm{~min}$, then processed directly for immunofluorescent labeling.

Antibodies and concentrations used were as follows: anti-GFIl at 1: 1000 to 1:2000 (Wallis et al. 2003), anti-NGN3 at 1:500 (a gift from Doris Stoffers, University of Pennsylvania, Philadelphia), anti-CgA at 1:2000 (ImmunoStar, Inc.), anti-Ki67 at 1:1000 (Novacastra Laboratories Ltd.) anti-BrdU at 1:50 (DakoCytomation), anti-muramidase/lysozyme at 
1:2000 to 1:10,000 (Novacastra Laboratories Ltd), anti-ATH-1 (MATH1) at 1:200 (Chemicon International), anti- $\beta$-galactosidase at 1:250 (Rockland), anti-neurotensin at 1:2000 (ImmunoStar), anti-NPY (which crossreacts with PYY) at 1:2000 (ImmunoStar), anti-secretin at 1:200 (Chemicon International), anti-CCK at 1:500 (ImmunoStar), anti-serotonin at 1:10,000 (ImmunoStar), and anti- $\beta$-galactosidase (1:250, Rockland). Apoptotic cells were identified with the Apoptag peroxidase kit (Chemicon).

\section{Quantification of cell numbers and statistical methods}

Images of alcian blue/nuclear fast red- or antibody/hematoxylin-stained tissue were acquired and counts of villus nuclei and alcian blue-positive or CgA-positive cells were performed. The percentage of alcian blue- or antigen-positive villus cells was determined for each intestinal region from each animal, and an average percentage for $\mathrm{Gfi1}^{+/-}$and $\mathrm{Gfi1}^{-/-}$tissues was determined. A standard deviation was calculated for each average and ANOVA and post-hoc $t$-tests were performed to measure statistical significance.

\section{Acknowledgments}

We thank Barbara Antalffy for assistance with histology; Karen L. Schulze for assistance with artwork; Stuart Orkin for providing $\mathrm{Gfi1}^{-/-}$mice; Doris Stoffers for the NGN3 antibody; and Susan J. Henning, Michael A. Helmrath, Christopher Dekaney, and members of the Zoghbi and Bellen laboratories for helpful discussions. This work was supported by grants from NASA and NIH (F32 DK63747, T32 DK07664, F32 DC5109, P30 HD24064, and P30 DK56338). H.Y.Z. and H.J.B. are investigators with the Howard Hughes Medical Institute.

\section{References}

Akazawa, C., Ishibashi, M., Shimizu, C., Nakanishi, S., and Kageyama, R. 1995. A mammalian helix-loop-helix factor structurally related to the product of Drosophila proneural gene atonal is a positive transcriptional regulator expressed in the developing nervous system. $J$. Biol. Chem. 270: 8730-8738.

Batlle, E., Henderson, J.T., Beghtel, H., van den Born, M.M., Sancho, E., Huls, G., Meeldijk, J., Robertson, J., van de Wetering, M., Pawson, T., et al. 2002. $\beta$-Catenin and TCF mediate cell positioning in the intestinal epithelium by controlling the expression of EphB/ephrinB. Cell 111: 251-263.

Beck, F., Chawengsaksophak, K., Waring, P., Playford, R.J., and Furness, J.B. 1999. Reprogramming of intestinal differentiation and intercalary regeneration in Cdx2 mutant mice. Proc. Natl. Acad. Sci. 96: 73187323.

Ben-Arie, N., Bellen, H.J., Armstrong, D.L., McCall, A.E., Gordadze, P.R., Guo, Q., Matzuk, M.M., and Zoghbi, H.Y. 1997. Math1 is essential for genesis of cerebellar granule neurons. Nature 390: 169-172.

Ben-Arie, N., Hassan, B.A., Bermingham, N.A., Malicki, D.M., Armstrong, D., Matzuk, M., Bellen, H.J., and Zoghbi, H.Y. 2000. Functional conservation of atonal and Math1 in the CNS and PNS. Development 127: 1039-1048.

Bermingham, N.A., Hassan, B.A., Price, S.D., Vollrath, M.A., Ben-Arie, N., Eatock, R.A., Bellen, H.J., Lysakowski, A., and Zoghbi, H.Y. 1999. Math1: An essential gene for the generation of inner ear hair cells. Science 284: 1837-1841.

Bermingham, N.A., Hassan, B.A., Wang, V.Y., Fernandez, M., Banfi, S., Bellen, H.J., Fritzsch, B., and Zoghbi, H.Y. 2001. Proprioceptor pathway development is dependent on Math1. Neuron 30: 411-422.

Cheng, H. and Leblond, C.P. 1974. Origin, differentiation and renewal of the four main epithelial cell types in the mouse small intestine. I. Columnar cell. Am. J. Anat. 141: 461-479.

Hock, H., Hamblen, M.J., Rooke, H.M., Traver, D., Bronson, R.T., Cameron, S., and Orkin, S.H. 2003. Intrinsic requirement for zinc finger transcription factor Gfi-1 in neutrophil differentiation. Immunity 18: 109-120.

Hock, H., Hamblen, M.J., Rooke, H.M., Schindler, J.W., Saleque, S., Fujiwara, Y., and Orkin, S.H. 2004. Gfi-1 restricts proliferation and preserves functional integrity of haematopoietic stem cells. Nature 431: 1002-1007.

Jafar-Nejad, H. and Bellen, H.J. 2004. Gfi/Pag-3/senseless zinc finger proteins: A unifying theme? Mol. Cell. Biol. 24: 8803-8812.

Jafar-Nejad, H., Acar, M., Nolo, R., Lacin, H., Pan, H., Parkhurst, S.M., and Bellen, H.J. 2003. Senseless acts as a binary switch during sensory organ precursor selection. Genes \& Dev. 17: 2966-2978.

Jenny, M., Uhl, C., Roche, C., Duluc, I., Guillermin, V., Guillemot, F., Jensen, J., Kedinger, M., and Gradwohl, G. 2002. Neurogenin3 is differentially required for endocrine cell fate specification in the intestinal and gastric epithelium. EMBO J. 21: 6338-6347.

Karsunky, H., Zeng, H., Schmidt, T., Zevnik, B., Kluge, R., Schmid, K.W., Duhrsen, U., and Moroy, T. 2002. Inflammatory reactions and severe neutropenia in mice lacking the transcriptional repressor Gfil. Nat. Genet. 30: 295-300.

Katz, J.P., Perreault, N., Goldstein, B.G., Lee, C.S., Labosky, P.A., Yang, V.W., and Kaestner, K.H. 2002. The zinc-finger transcription factor Klf4 is required for terminal differentiation of goblet cells in the colon. Development 129: 2619-2628.

Kazanjian, A., Wallis, D., Au, N., Nigam, R., Venken, K.J., Cagle, P.T., Dickey, B.F., Bellen, H.J., Gilks, C.B., and Grimes, H.L. 2004. Growth factor independence-1 is expressed in primary human neuroendocrine lung carcinomas and mediates the differentiation of murine pulmonary neuroendocrine cells. Cancer Res. 64: 6874-6882.

Korinek, V., Barker, N., Moerer, P., van Donselaar, E., Huls, G., Peters, P.J., and Clevers, H. 1998. Depletion of epithelial stem-cell compartments in the small intestine of mice lacking Tcf-4. Nat. Genet. 19: 379-383.

Madara, J.L. and Trier, J.S. 1994. The functional morphology of the mucosa of the small intestine. In Physiology of the gastrointestinal tract (ed. L.R. Johnson), pp. 1577-1622. Raven Press, New York.

Mutoh, H., Hakamata, Y., Sato, K., Eda, A., Yanaka, I., Honda, S., Osawa, H., Kaneko, Y., and Sugano, K. 2002. Conversion of gastric mucosa to intestinal metaplasia in Cdx2-expressing transgenic mice. Biochem. Biophys. Res. Commun. 294: 470-479.

Okubo, T. and Hogan, B.L. 2004. Hyperactive Wnt signaling changes the developmental potential of embryonic lung endoderm. J. Biol. 3: 11.

Pinto, D., Gregorieff, A., Begthel, H., and Clevers, H. 2003. Canonical Wnt signals are essential for homeostasis of the intestinal epithelium. Genes \& Dev. 17: 1709-1713.

Sancho, E., Batlle, E., and Clevers, H. 2004. Signaling pathways in intestinal development and cancer. Annu. Rev. Cell Dev. Biol. 20: 695723.

Schmidt, T., Zornig, M., Beneke, R., and Moroy, T. 1996. MoMuLV proviral integrations identified by Sup-F selection in tumors from infected myc/pim bitransgenic mice correlate with activation of the gfi-1 gene. Nucleic Acids Res. 24: 2528-2534.

Schonhoff, S.E., Giel-Moloney, M., and Leiter, A.B. 2004. Minireview: Development and differentiation of gut endocrine cells. Endocrinology 145: 2639-2644.

Soubeyran, P., Andre, F., Lissitzky, J.C., Mallo, G.V., Moucadel, V., Roccabianca, M., Rechreche, H., Marvaldi, J., Dikic, I., Dagorn, J.C., et al. 1999. Cdx1 promotes differentiation in a rat intestinal epithelial cell line. Gastroenterology 117: 1326-1338.

van de Wetering, M., Sancho, E., Verweij, C., de Lau, W., Oving, I., Hurlstone, A., van der Horn, K., Batlle, E., Coudreuse, D., Haramis, A.P., et al. 2002. The $\beta$-catenin/TCF-4 complex imposes a crypt progenitor phenotype on colorectal cancer cells. Cell 111: 241-250.

van Es, J.H., Jay, P., Gregorieff, A., van Gijn, M.E., Jonkheer, S., Hatzis, P., Thiele, A., van den Born, M., Begthel, H., Brabletz, T., et al. 2005. Wnt signalling induces maturation of Paneth cells in intestinal crypts. Nat. Cell Biol. 7: 381-386.

Wallis, D., Hamblen, M., Zhou, Y., Venken, K.J., Schumacher, A., Grimes, H.L., Zoghbi, H.Y., Orkin, S.H., and Bellen, H.J. 2003. The zinc finger transcription factor Gfil, implicated in lymphomagenesis, is required for inner ear hair cell differentiation and survival. Development 130: 221-232.

Yang, Q., Bermingham, N.A., Finegold, M.J., and Zoghbi, H.Y. 2001. Requirement of Math1 for secretory cell lineage commitment in the mouse intestine. Science 294: 2155-2158. 


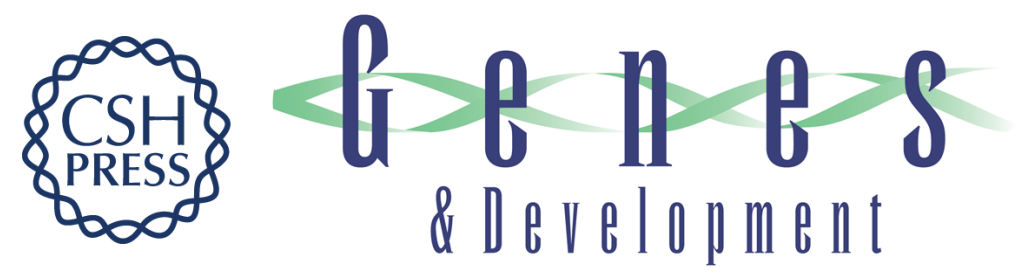

\section{Gfi1 functions downstream of Math1 to control intestinal secretory cell subtype allocation and differentiation}

Noah F. Shroyer, Deeann Wallis, Koen J.T. Venken, et al.

Genes Dev. 2005, 19:

Access the most recent version at doi:10.1101/gad.1353905

Supplemental
Material http://genesdev.cshlp.org/content/suppl/2005/09/29/19.20.2412.DC1

References This article cites 28 articles, 12 of which can be accessed free at:

http://genesdev.cshlp.org/content/19/20/2412.full.html\#ref-list-1

License

Email Alerting

Receive free email alerts when new articles cite this article - sign up in the box at the top

Service

right corner of the article or click here.

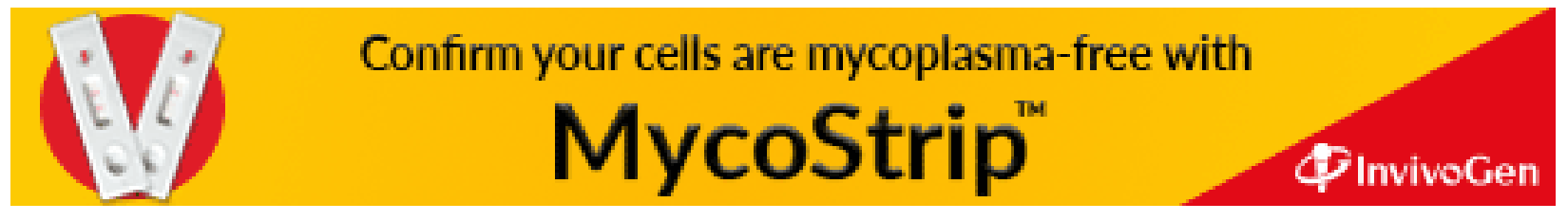

